\title{
Development of TES-based detectors array for the X-ray Integral Field Unit (X-IFU) on the future X-Ray Observatory Athena.
}

L. Gottardi ${ }^{a}$, H. Akamatsu ${ }^{a}$, D. Barret ${ }^{b}$, M.P. Bruijn ${ }^{a}$, R.H. den $\operatorname{Hartog}^{a}$, J-W. den $\operatorname{Herder}^{a}$, H.F.C. Hoevers ${ }^{a}$, M. Kiviranta ${ }^{c}$, J. van der Kuur ${ }^{a}$, A.J. van der Linden ${ }^{a}$, B.D. Jackson ${ }^{a}$, M. Jambunathan $^{a}$, M.L. Ridder ${ }^{a}$

${ }^{a}$ SRON National Institute for Space Research, Sorbonnelaan 2, 3584 CA Utrecht, The Netherlands

${ }^{b}$ Institut de Recherche en Astrophysique et Plantologie (IRAP), Avenue du Colonel Roche.BP 44346, 31028 Toulouse Cedex 4, France

${ }^{c} \mathrm{VTT}$

Tietotie 3, 02150 Espoo, Finland

\begin{abstract}
We are developing transition-edge sensor (TES)-based microcalorimeters for the X-ray Integral Field Unit (XIFU) of the future European X-Ray Observatory Athena. The microcalorimeters are based on TiAu TESs coupled to $250 \mu \mathrm{m}$ squared, AuBi absorbers. We designed and fabricated devices with different contact geometries between the absorber and the TES to optimise the detector performance and with different wiring topology to mitigate the self-magnetic field. The design is tailored to optimise the performance under Frequency Domain Multiplexing. In this paper we review the main design feature of the pixels array and we report on the performance of the 18 channels, $2-5 \mathrm{MHz}$ frequency domain multiplexer that will be used to characterised the detector array.
\end{abstract}

Keywords: X-IFU,Athena, FDM, cryogenic detector,TES, SQUID, x-ray microcalorimeter

\section{INTRODUCTION}

Transition-edge sensor (TES)-based microcalorimeters are the chosen technology for the detectors array of the X-ray Integral Field Unit (X-IFU) on board of the future European X-Ray Observatory Athena. The X-IFU is a 2-D imaging integral-field spectrometer operating in the soft X-ray band $(0.3-12 \mathrm{keV})$. The detector consists of an array of 3840 TESs coupled to X-ray absorbers and read out using Frequency Domain Multiplexing (FDM) in the $\mathrm{MHz}$ bandwidth. The Athena proposed design calls for devices with an high filling-factor, high quantumefficiency, high count-rate capability and an energy resolution of $2.5 \mathrm{eV}$ at $5.9 \mathrm{keV}$.

The requirements for the most relevant pixel-related parameters for the Athena X-IFU instrument are listed in Tab. (1)

\begin{tabular}{ll}
\hline Parameters & Requirements \\
\hline Energy range & $0.2-12 \mathrm{keV}$ \\
Energy resolution: $E<7 \mathrm{keV}$ & $2.5 \mathrm{eV}$ \\
Pixel size & $\left.250 \times 250 \mu \mathrm{m}^{2}\right)$ \\
Field of view & $5^{\prime}$ (diameter $)(3840$ pixels $)$ \\
Quantum efficiency @ $6 \mathrm{keV}$ & $>90 \%$ \\
Count rate capability - faint source & $1 \mathrm{mCrab}(>80 \%$ high-resolution events $)$ \\
Count rate capability - bright source & 1 Crab $(>30 \%$ low-resolution events $)$ \\
\hline
\end{tabular}

Table 1. Key performance requirements for the Athena X-IFU pixels array 
At SRON we develop microcalorimeters arrays based on superconducting TiAu bilayers TES deposited on suspended SiN membranes thermally coupled to $\mathrm{Cu} / \mathrm{Bi}$ film absorbers. The Bismuth layer is added on top of the $\mathrm{Cu}$ layer to achieve sufficient stopping power without increasing significantly the total absorber heat capacity. In the past we have shown that pixels made of TiAu TESs coupled to a $1 \mu \mathrm{m}$-thick $\mathrm{Cu}$ and $2.6 \mu \mathrm{m}$-thick Bi $100 \times 100 \mu \mathrm{m}^{2}$ central absorber could achieve high energy resolution ${ }^{1}$ when DC biased. The K-alpha spectrum of a Fe-55 radioactive source detected with this pixel is shown in Fig. (10). We measured an energy resolution of $2.5 \mathrm{eV}$ at $5.9 \mathrm{keV}$ under DC bias.

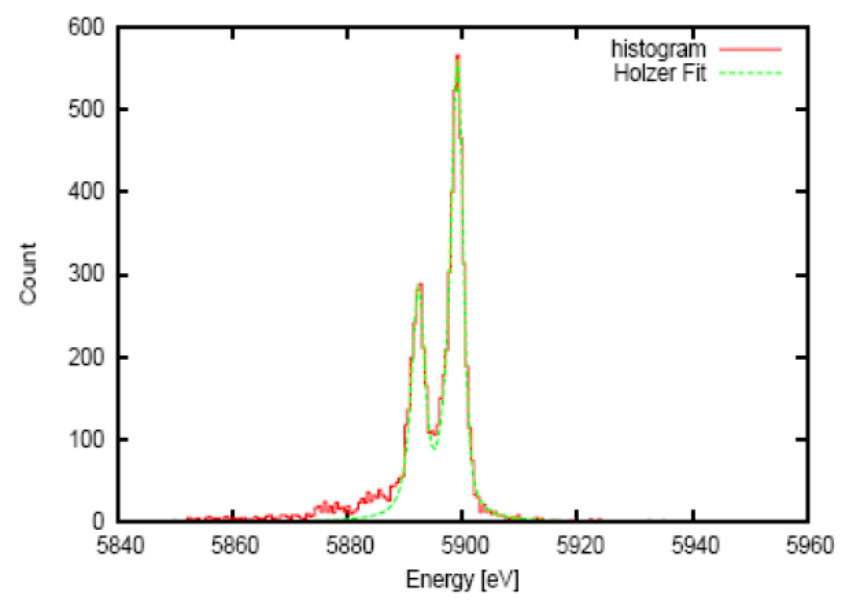

Figure 1. ${ }^{55} \mathrm{Fe}$ spectrum taken with a $100 \times 100 \mu \mathrm{m}^{2}, 1 \mu \mathrm{m}-$ thick $\mathrm{Cu}$ and $2.6 \mu \mathrm{m}-$ thick Bi absorber.

An identical pixel with central $\mathrm{Cu}$ absorber has been extensively characterised in a syncrotron facility with X-ray photons with energy ranging from 150 to $2000 \mathrm{eV}$ [2] We demonstrated an high X-ray energy resolution and high count-rate capability as shown in Fig. (2), where the energy resolution as a function of the incident photon as well as the energy spectrum for a photon flux of about 500 photons/sec are plotted. At $250 \mathrm{eV}$

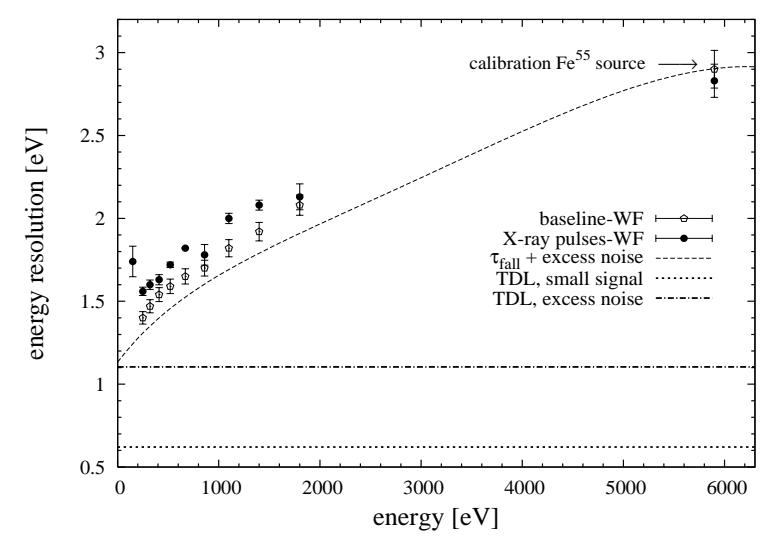

a)

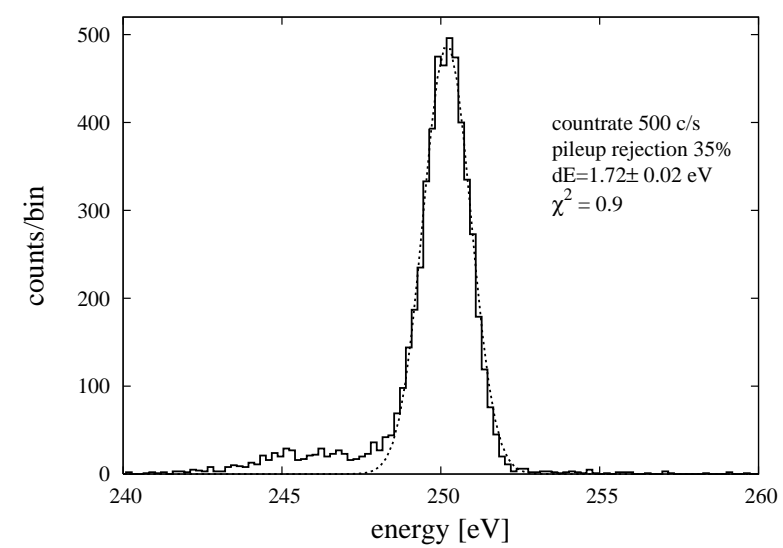

b)

Figure 2. (a.) Energy resolution as a function of incident photon energy for a pixel with $100 \times 100 \mu \mathrm{m}^{2}, 1 \mu$-thick Cu absorber. (b.) Energy spectrum at $250 \mathrm{eV}$ for a photon flux of about 500 photons/sec.

and low flux rate we measured the best energy resolution of $1.52 \pm 0.02 \mathrm{eV}$, which only slightly degraded to $1.78 \pm 0.02 \mathrm{eV}$ with 500 photons/sec hitting the detector. 2. This pixel has been fully characterised under AC bias as well and we reported a best energy resolution of $3.7 \mathrm{eV}$ ! The performance of these pixels is however limited by a poor filling factor (about 20\%), and a relatively low absorption efficiency, where a maximum of $74 \%$ at $5.9 \mathrm{keV}$ with a $2.6 \mu \mathrm{m}$-thick Bi layer has been achieved. Moreover they have never been tested so far with a Frequency Domain Multiplexer operating at $\mathrm{MHz}$ frequencies. The most representative results of a $\mathrm{MHz}$ 
Frequency Domain Multiplexing (FDM) read-out of X-IFU-like TES microcalorimeters is reviewed by Ravera et al,$\frac{5}{5}$ where it is shown that an energy resolution of $3.6 \mathrm{eV}$ at $5.9 \mathrm{keV}$ has been demonstrated ${ }^{\sqrt{6}}$ using detectors fabricated at Goddard 7

We have recently started a new development program aiming at the fabrication of large array detectors, which will meet the X-IFU instrument requirements when operating with the $\mathrm{MHz}$-FDM read-out. In the following session we present the current status of the fabrication of arrays with large $\mathrm{CuBi}$ and $\mathrm{AuBi}$ absorber and show the performance of the FDM set-up that will be used for the single pixel characterisation.

\section{MICROCALORIMETER ARRAY FABRICATION}

Here we highlight the improvements made in the process fabrication of the micro-calorimeter array. The renewed mask design consists of devices focusing on absorber coupling, alpha tuning, coplanar/strip line wiring configuration and self-magnetic field mitigation wiring topology.

Earlier experiments showed that, by optimising the image reversal resist, Bi deposition and liftoff procedure, a free hanging mushroom absorber with only one stem attached to the TES for the thermal contact and 4 supporting ones to the substrate can be fabricated. Fig. (3) shows optical images of test pixels from a $40 \times 40$ array where the absorber is supported from the substrate by 4 stems with a diameter size of $5 \mu \mathrm{m}$.

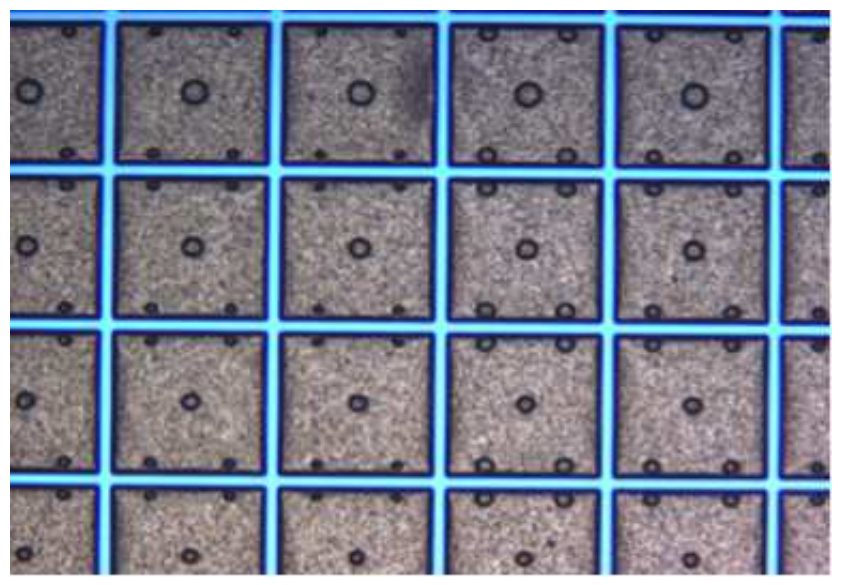

a)

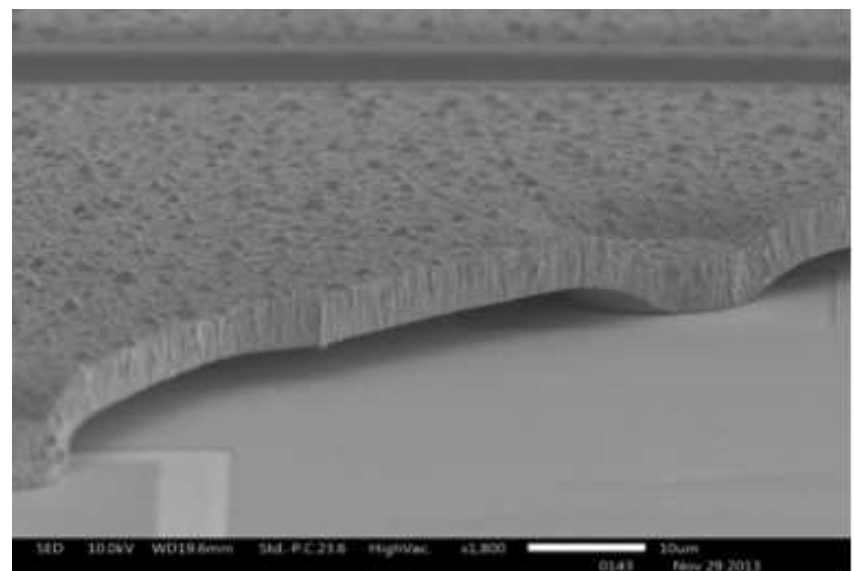

b)

Figure 3. (a.) Optical image of a 40x40 pixel array with $240 \times 240 \mu \mathrm{m}^{2}$ Bi absorbers.(b.) SEM image of a mushroom shaped $\mathrm{Bi}$ absorber hat connected to the $\mathrm{Au}$ TES at the stem regions.

In order to provide a weak thermal link to the cooling bath, the calorimeter must be fabricated on the silicon nitride membrane unlike solid silicon substrate. Recently, we have fabricated the TES and the wiring layer on the SiN membrane. SiN slots and Cu dots fabrication( for alpha tuning) is also implemented as shown in Fig. (4).

The current work will be finalised by fabricating the mushroom shaped $\mathrm{Cu}$-Bi absorber hat with foot region on the SiN membrane to demonstrate the absorber coupling. The future area of focus will be in developing the electroplated $\mathrm{Au}$ process to replace the $\mathrm{Cu}$ in the absorber for a further optimisation of the pixel performance according to the X-IFU requirements.

\section{EXPERIMENTAL SET-UP}

An FDM multiplexer similar to the one described by Gottardi et al ${ }^{8}$ is mounted on a removable probe of a helium-free dilution refrigerator from Leiden Cryogenics. It consists of a low magnetic impurity copper bracket mounting on top a two-stage SQUID amplifier, the LC filters and the TES array chips and a printed circuit board for the electrical connection. One thermometer and one heater are glued at the bottom of the copper plate for the read-out and stabilisation of the temperature. The FDM set-up can be used both with ultra-low noise equivalent power (NEP) TES bolometers and with high energy resolving power X-ray microcalorimeters. 


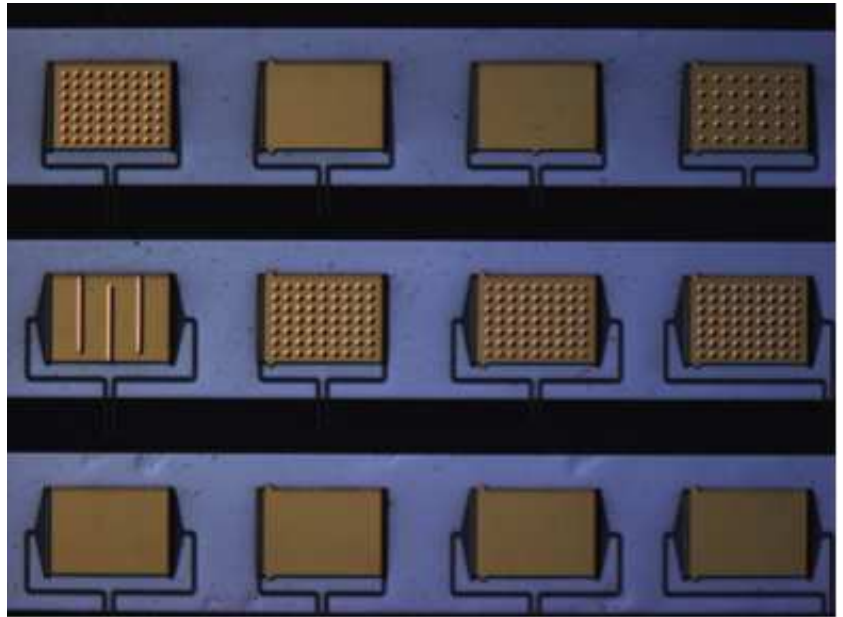

a)

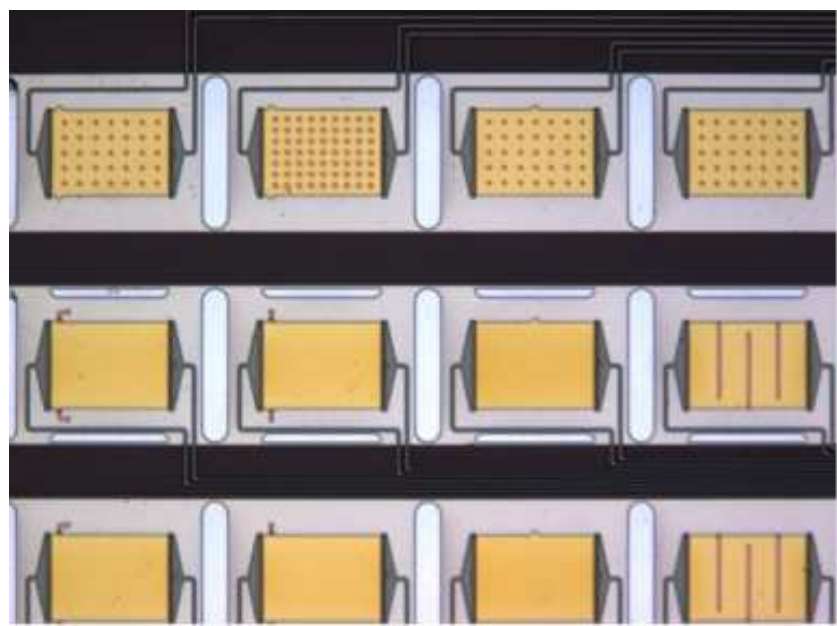

b)

Figure 4. (a.) Optical image of micro-calorimeter $5 \times 5$ array with TES, wiring and Cu dots on a SiN membrane. (b.) Optical image of the SiN slots on the micro-calorimeter array.

The former requires very low parasitic power loading $(<1 \mathrm{fW})$, which is achieved by means of light blocking filters in the signal loom feedthrough and a light-tight assembly. The X-ray microcalorimeters are very sensitive to magnetic fields and their performance is optimal at static field lower than $1 \mu$ Tesla). Special care has been taken to design the magnetic shielding and to improve the uniformity of the applied magnetic field across the array. The TES arrays chip fits into a superconducting Helmholtz coil fixed at one end of the bracket. The coil is used to generate an uniform perpendicular magnetic field over the whole pixels array. The circuitry PC-board currently allows to read-out 18 pixels under ac bias in a FDM configuration. The SQUID chip is fixed to the copper bracket using high thermal conductivity glue (Ge Varnish). The TES array chip is connected via a superconducting interconnection chip to the lithographic high-Q LC resonators arrays developed at SRON 91 The nominal inductance of the coil used in each filter is $L=400 \mathrm{nH}$, while the capacitance's $\mathrm{C}$ are designed such that the frequencies $f_{0}=\frac{1}{2 \pi \sqrt{L C}}$ are spread at a constant interval of about $200 \mathrm{kHz}$ in the range from 2 to $5 \mathrm{MHz}$. The main purpose of the FDM test facility described here is to increase the experimental throughput for single pixel characterization. The FDM demonstrator for the Athena X-IFU instrument is described elsewhere ${ }^{10}$.

The shielding of the external magnetic field is achieved by inserting the probe hosting the FDM set-up into a double-shield configuration made of cryoperm and lead. can wrapped by few layers of metallic glass tape.

For the FDM read-out we use a low noise two-stage SQUID array provided by VTT. We use a VTT $180 \times 4$ SQUIDs array as amplifier SQUID and a low input inductance VTT 6-subloop fractional-turn SQUID as the front-end SQUID connected to the LC resonators and the TES array 11 The front-end SQUID is voltage bias by applying a DC bias current to a $1 \Omega$ shunt resistor connected in series between the lower-SQUID output to the upper-SQUID input coil. Both SQUID arrays can be operated in the standard not-linearized mode or in the current sensing mode with on chip linearization.12 The SQUID chips are thermally coupled to the bracket by means of several Au bonding.

The SQUIDs array signal is read out using low-noise room temperature FEE developed at SRON operating both in open-loop mode or in Baseband Feedback mode! 13

\section{FDM READ-OUT CHARACTERISATION}

Here we present the results of the two-stage SQUID characterisation at $T=25 \mathrm{mK}$ without LC resonators and TES microcalorimeters connected to the front-end SQUID input coil.

In Fig. (5) we show the voltage-to-flux characteristics of the two-stage SQUIDs measured as a function of the bias current of the front-end SQUID. The two-stage SQUIDs array shows clean V- $\Phi$ curves as well with a maximum voltage modulation of $2.4 \mathrm{mV}$ at a lower-SQUID bias current of $50 \mu \mathrm{A}$. 


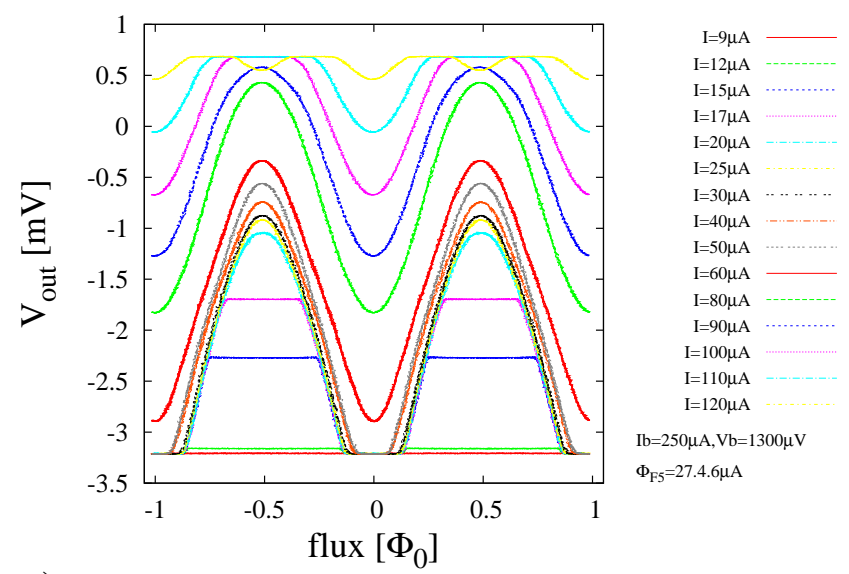

a)

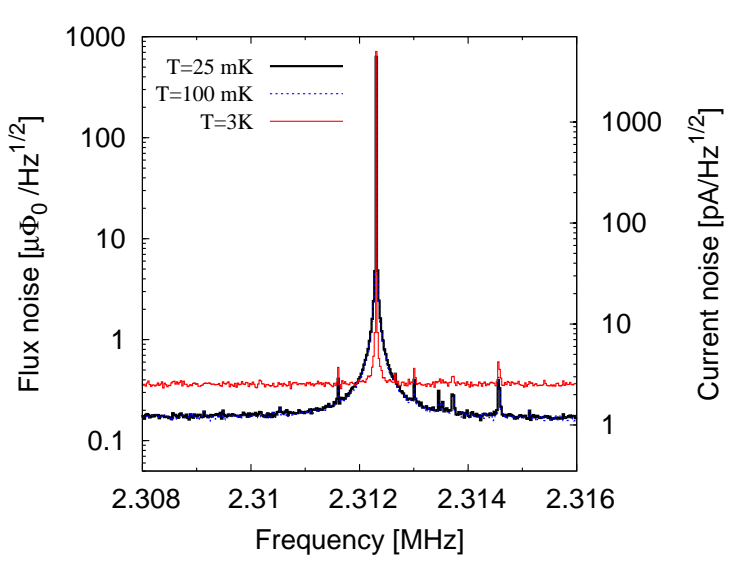

b)

Figure 5. (a.) Voltage-to-flux characteristics of the two-stage SQUIDs configuration operating at 20mK. (b.) Flux and current noise spectra density for the VTT two-stage SQUIDs measured at at $3 \mathrm{~K}$ and $20 \mathrm{mK}$ with a calibration signal at $2.3 \mathrm{MHz}$.

We measured the current noise of the two-stage SQUID at three different temperature, respectively $3 \mathrm{~K}, 100 \mathrm{mK}$ and $20 \mathrm{mK}$. Two calibration tones at $12 \mathrm{kHz}$ and $2 \mathrm{MHz}$ have been applied via the feedback coil to monitor the SQUID gain as a function of temperature. The magnitude of the calibration tone correspond to an applied flux to the lower SQUID of $0.01 \Phi_{0}$. In Fig. (5).a the spectral flux and current density taken at temperature of $3 \mathrm{~K}$ and $25 \mathrm{mK}$ is shown. The current noise has been calculate using the nominal input sensitivity of the front-end SQUID array equal to $M^{-1}=7 \mu \mathrm{A} / \Phi_{0}$ In Fig. (5).b a zoom-in of the SQUID noise spectra around the $2 \mathrm{MHz}$ calibration tone is shown.

We measured a flux noise equal to $3.5 \cdot 10^{-7} \Phi_{0} / H z^{1 / 2}$ and $1.8 \cdot 10^{-7} \Phi_{0} / H z^{1 / 2}$ at $3 \mathrm{~K}$ and $20 \mathrm{mK}$ respectively. This corresponds to an energy resolution of $\epsilon=S_{\Phi} / 2 L_{s q} \simeq 9 \hbar$, where an $L_{s q}=70 \mathrm{pH}$ is assumed. We believe the measured noise is limited by the room temperature amplifier and the read-out acquisition system. A relatively straight forward optimisation of the room temperature set-up should lead to a further improvement of the SQUID sensitivity. The quantum limited flux sensitivity for the front-end SQUID array is $\sqrt{S_{\Phi, Q L}}=6 \cdot 10^{-8} \Phi_{0} / H z^{1 / 2}$, which should be achievable if the temperature of the SQUID shunt resistors can be cooled at temperature below $150 \mathrm{mK}$. The measured current noise at the SQUID input is $1.2 \cdot 10^{-12} \mathrm{~A} / \mathrm{Hz} z^{1 / 2}$ at $20 \mathrm{mK}$, which corresponds to a coupled energy resolution of $\epsilon_{i i}=\frac{L_{i n}}{2} S_{i i} \sim 20 \hbar$, where $L_{i n} \sim 3 \mathrm{nH}$ is the input inductance of the SQUID. The two-stage SQUID was then tested in combination with 18 high- $Q L C$ resonators and an array of TES-bolometers. We measured a SQUID input current noise of $3 \mathrm{pA} / \sqrt{\mathrm{Hz}}$. The contribution of the SQUID noise on the energy resolution for a single pixel read-out has been estimated to be better than $0.1 \mathrm{eV}$. The combination of low input inductance and low input current noise of the front-end SQUID is essential to achieve a high multiplexing factor in the FDM system proposed for Athena $\underline{10}$

In Fig. (6) we show the SQUID response of a frequency sweep across the resonators via the ac-bias with the TESs in superconducting and in the normal state respectively.

\section{CONCLUSION}

We fabricated and tested an FDM set-up for the single pixel characterisation of TES based microcalorimeters under ac bias in the $\mathrm{MHz}$ frequency range. The set-up is designed to read out 18 pixels under ac bias and 2 pixels under AC or DC bias.

We validate the performance of the ac bias read-out by characterising a the novel VTT two-stage SQUID amplifier and the lithographic high- $Q L C$ filters at $T=25 \mathrm{mK}$ in a removable probe of a He-free dilution refrigerator from Leiden Cryogenics. The two-stage SQUID amplifier developed at VTT has a measured flux noise at $25 \mathrm{mK}$ equal to $1.8 \cdot 10^{-7} \Phi_{0} / H z^{1 / 2}$ and an input current noise of $1.2 \cdot 10^{-12} \mathrm{~A} / \mathrm{Hz} z^{1 / 2}$, which corresponds to a coupled energy resolution of $\epsilon_{i i}=20 \hbar$ over the whole interesting frequency range from 2 to $5 \mathrm{MHz}$. 


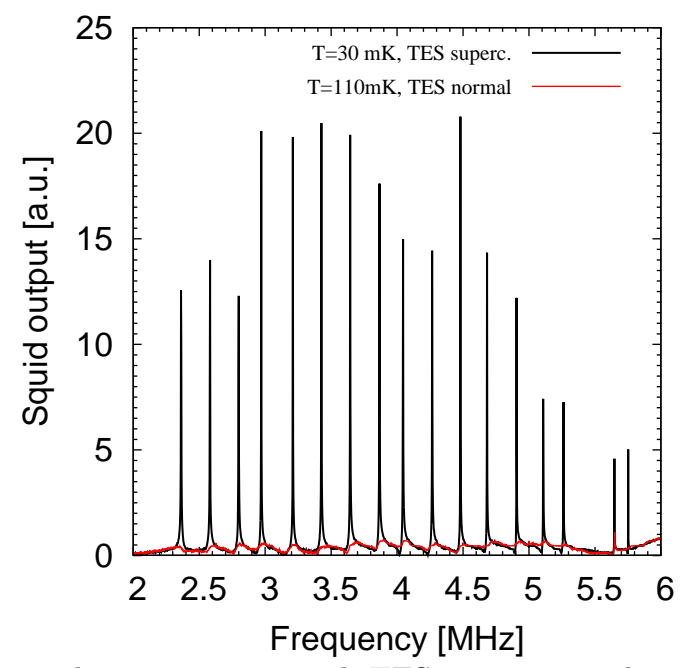

Figure 6. Frequency sweep via the ac-bias input port with TESs in superconducting (black) and normal (red) state.

We have shown the progress in the fabrication of TES microcalorimeter array based on TiAu TESs and large $\mathrm{CuBi}$ absorber. The pixels have been designed to meet the requirements of the X-IFU instrument on board of Athena.

\section{REFERENCES}

1. Dirks, B., Popescu, M., Bruijn, M., Gottardi, L., Hoevers, H., de Korte, P., van der, K., and J. Ridder, M., "TiAu-based micro-calorimeters for space applications," Nuclear Instruments and Methods in Physics Research Section A: Accelerators, Spectrometers, Detectors and Associated Equipment 610, 83-86 (2009).

2. Gottardi, L., Takei, Y., Kuur van der, J., Korte de, P., Hoevers, H., Boersma, D., Bruijn, M., Mels, W., Ridder, M., Takken, D., and Weers van, H., "Characterisation of a TES-based X-ray microcalorimeter in the energy range from 150 to $1800 \mathrm{eV}$ using an adiabatic demagnetisation regrigerator," Journal of Low Temperature Physics 151, 106-111 (2008).

3. Takei, Y., Gottardi, L., Kuur van der, J., Korte de, P., Hoevers, H., Boersma, D., Bruijn, M., Mels, W., Ridder, M., Takken, D., and Weers van, H., "Characterization of a high-performance Ti/Au TES microcalorimeter with a central Cu absorber," Journal of Low Temperature Physics 151, 500-505 (2008).

4. van der Kuur, J., Gottardi, L., Borderias, M., Dirks, B., de Korte, P., Lindeman, M., Khosropanah, P., den Hartog, R., and Hoevers, H., "Performance of an x-ray single pixel TES microcalorimeter under DC and AC biasing," AIP COnference Proceedings 1185, 538-541 (2009).

5. Ravera, L., et al.., "The X-ray Integral Field Unit (X-IFU) for Athena," This Proceedings (2014).

6. Akamatsu, H., Gottardi, L., Adams, J., Bandler, S., Bruijn, . M., Chervenak, J., Eckart, M., Finkbeiner, F., Hartog, R., Hoevẽrs, H., Kelley, R., Kilbourne, C., Kuur, J., Linden, A., Portẽr, F., Sadleir, J., Smith, S., and Kiviranta, M., "Performance of TES X-ray microcalorimeters with ac bias read-out at MHz frequencies," Journal of Low Temperature Physics , 1-6 (2014).

7. Iyomoto, N., Bandler, S., Brekosky, R., Brown, A.-D., Chervenak, J., Figueroa-Feliciano, E., Finkbeiner, F., Kelley, R.L. Kilbourne, C., Porter, F., and Sadleir, J.E.and Smith, S., "Close-packed arrays of transitionedge x-ray microcalorimeters with high spectral resolution at $6 \mathrm{keV}, "$ Appl. Phys. Lett. 92, 13508 (2008).

8. Gottardi, L., Bruijn, M., Gao, J.-R., den Hartog, R., Hijmering, R., Hoevers, H., Khosropanah, P., de Korte, P., van der Kuur, J., Lindeman, M., and Ridder, M., "AC bias characterization of low noise bolometers for SAFARI using an open-loop frequency domain SQUID-based multiplexer operating between 1 and $5 \mathrm{MHz}$," Journal of Low Temperature Physics 167, 161-167 (2012).

9. Bruijn, M., Gottardi, L., den Hartog, R., Hoevers, H., Kiviranta, M., de Korte, P., and van der Kuur, J., "High-Q LC filters for FDM read out of cryogenic sensor arrays," Journal of Low Temperature Physics 167, 695-700 (2012). 
10. den Hartog, R., et al.., "Requirements for the read-out of the detector system of Athena X-IFU," This Proceedings (2014).

11. Kiviranta, M., Groenberg, L., Beev, N., and van der Kuur, J., "Some phenomena due to SQUID input properties when local feedback is present," EUCAS Proceedings (2013).

12. Kiviranta, M., "SQUID linearization by current-sampling feedback," Superconductor Science and Technology 21(4), 045009 (2008).

13. den Hartog, R., Audley, M., Beyer, J., Boersma, D., Bruijn, M., Gottardi, L., Hoevers, H., Hou, R., Keizer, G., Khosropanah, P., Kiviranta, M., de Korte, P., van der Kuur, J., van Leeuwen, B.-J., Nieuwenhuizen, A., and van Winden, P., "Low-noise readout of TES detectors with baseband feedback frequency domain multiplexing," Journal of Low Temperature Physics 167, 652-657 (2012). 

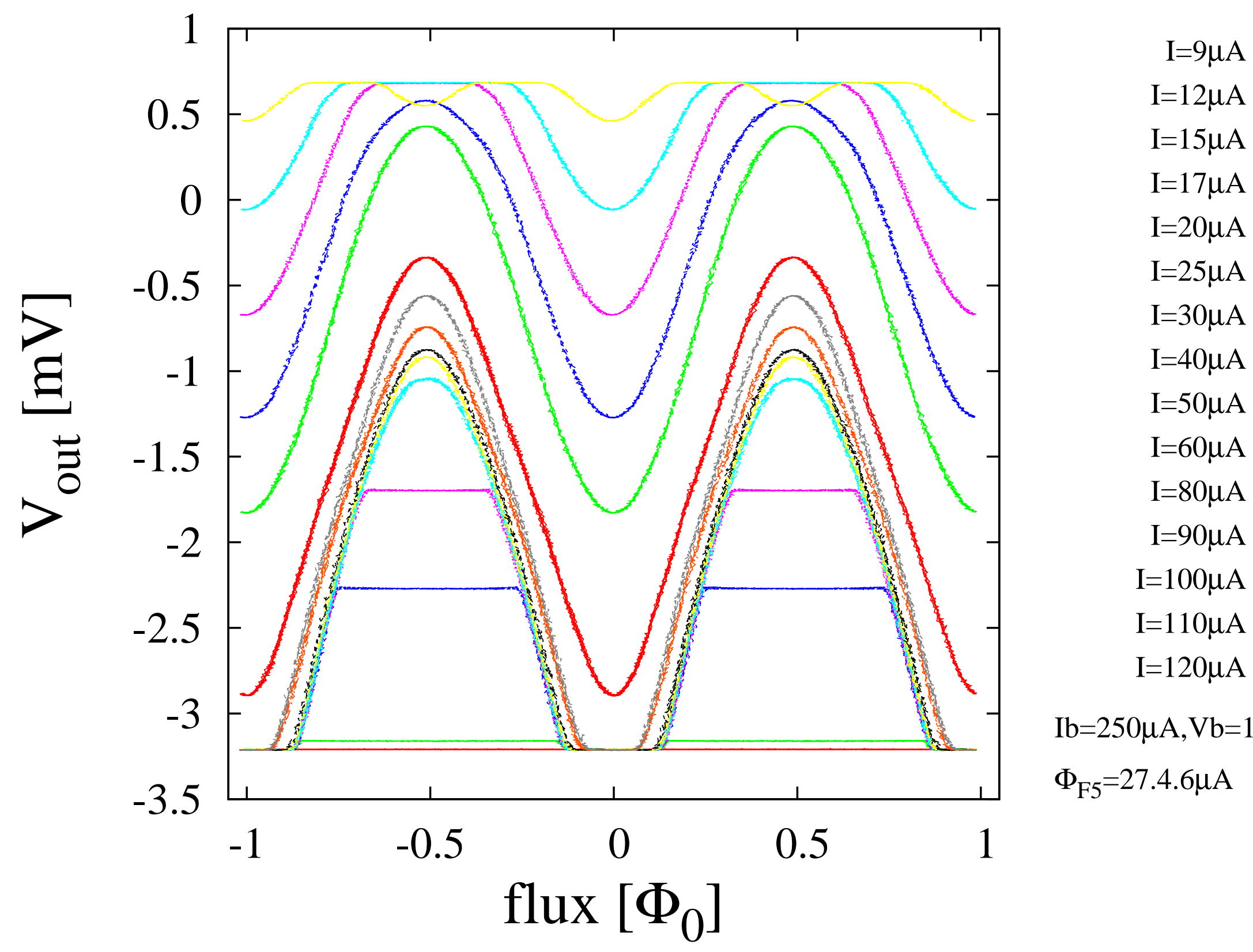

$\mathrm{Ib}=250 \mu \mathrm{A}, \mathrm{Vb}=1300 \mu \mathrm{V}$ $\Phi_{\mathrm{F} 5}=27.4 .6 \mu \mathrm{A}$ 


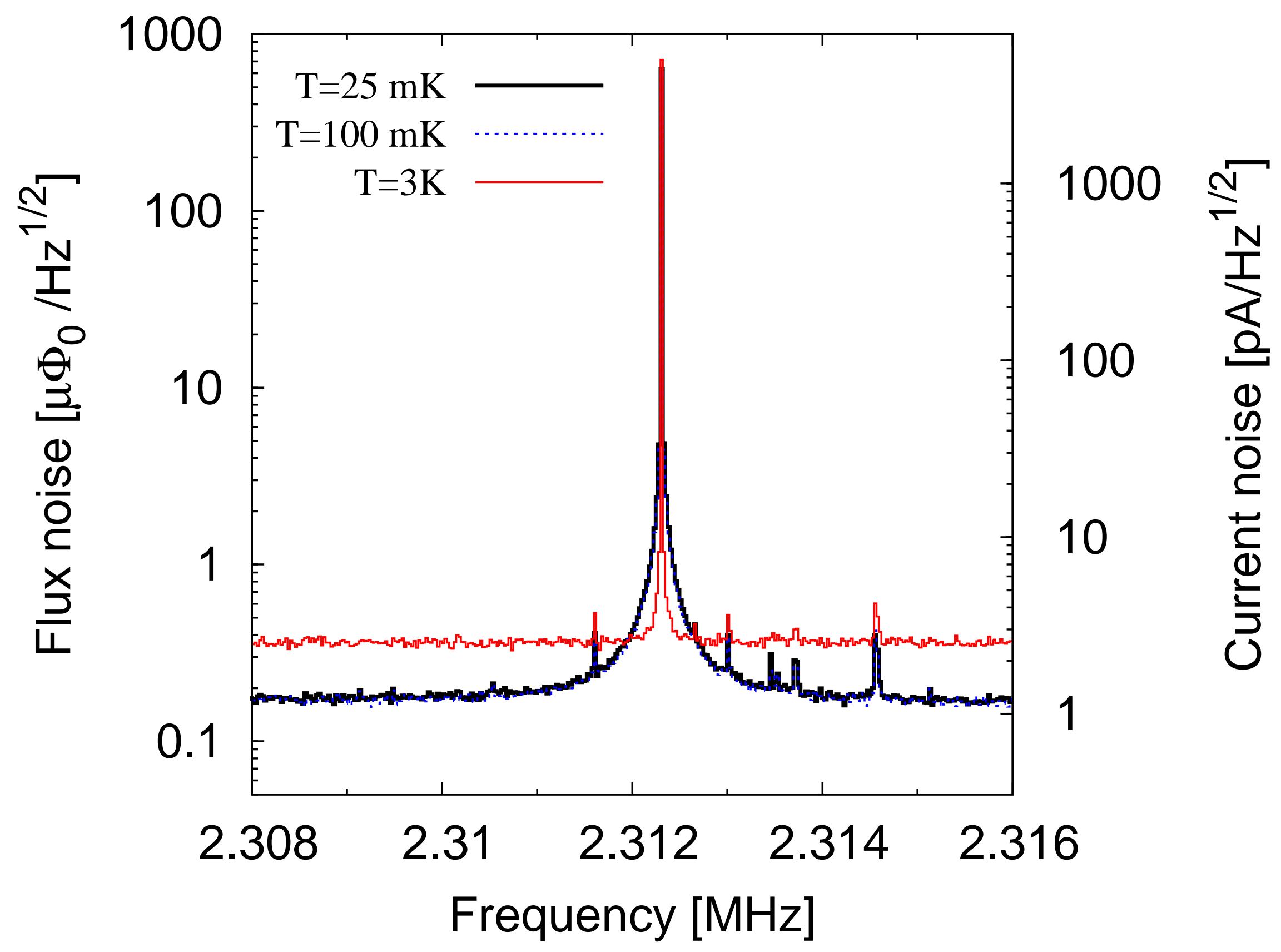

